\title{
Tangence
}

\section{L'écriture d'une allophone d'origine française}

\section{Régine Robin}

Numéro 59, janvier 1999

Écrivains d'ailleurs

URI : https://id.erudit.org/iderudit/025989ar

DOI : https://doi.org/10.7202/025989ar

Aller au sommaire du numéro

Éditeur(s)

Tangence

ISSN

0226-9554 (imprimé)

1710-0305 (numérique)

Découvrir la revue

Citer cet article

Robin, R. (1999). L'écriture d'une allophone d'origine française. Tangence, (59),

26-37. https://doi.org/10.7202/025989ar d'utilisation que vous pouvez consulter en ligne.

https://apropos.erudit.org/fr/usagers/politique-dutilisation/ 


\section{L'écriture d'une allophone d'origine française Régine Robin}

On me permettra de commencer par un souvenir, une anecdote. Lors d'un forum réunissant divers écrivains, un de ces forums comme il y en a tant au Québec, un de nos écrivains venu d'ailleurs mais parfaitement intégré dans la société québécoise dit à un moment donné, en substance: "Ici, la notion d'étranger n'existe pas". Je ne sais à quoi il faisait allusion, mais j'ai pris très au sérieux sa remarque. Il est vrai que le débat ne passe jamais par cette notion, pas plus qu'il ne passe par celle "d'immigrés" comme en France. La distinction s'établit ici entre les gens "de souche" et les autres, les "Néos". C'est à cette distinction que je voudrais m'attarder.

\section{Écrivain migrant, écrivain néo-québécois?}

Il y a un paradoxe dans l'institution littéraire québécoise. Le Québec en effet, rejette massivement la politique du multiculturalisme canadien. L'assimilation de type français (intrégration des individus et non des communautés) étant impossible en Amérique du Nord, on se trouve au Québec à promouvoir l'interculturel qui conjugue souvent (pas toujours) le meilleur et le pire des systèmes français et américains. La littérature au Québec n’est pas une littérature à trait d'union comme elle l'est dans des sociétés de plus en plus structurées selon des lignes ethniques. On a forgé un nouveau mot pour désigner les écrits et fictions d'auteurs qui viennent d'ailleurs. On dira littérature néo-québécoise ou encore romanciers, poètes ou dramaturges immigrés. Cette littérature ne vit pas en marge de l'institution littéraire, mais la désignation de "néo-québécois" constitue un marquage et est l'indice d'un problème. En témoigne par exemple la collection des Éditions Balzac, "Autres rives", lancée en 1994 avec deux auteurs bien connus dans le milieu: Fulvio Caccia avec Golden Eighties et Gérard Étienne avec Le nègre crucifié. On peut lire dans le manifeste de lancement de la collection:

Italiens, Grecs, Portugais, en ce qui a trait aux Européens, Asiatiques, Haïtiens, Juifs sépharades, les diverses minorités qui composent le Québec moderne, expriment, par un vécu et une histoire propres, une sensibilité quelque peu différente des 
Québécois de souche. C'est cette sensibilité que les Éditions Balzac veulent capter en ouvrant la toute nouvelle collection Autres rives.

C'est cette sensibilité qu'ont su capter les éditeurs du Canada anglais et qui a bouleversé et enrichí leur littérature... Au Québec, au contact d'une culture propre, les écrivains haïtiens, italiens ou autres, qui se sont forgés une écriture particulière, sont le reflet de cette symbiose entre la culture propre à l'émigré et celle de la terre d'adoption... ${ }^{1}$

Nombre d'auteurs du Québec sont ainsi des écrivains néoquébécois ${ }^{2}$.

Il faudrait pouvoir tous les citer. Ils sont d'origines les plus diverses, venant des quatre coins du monde et développant des

1 Le point d'ironie, bulletin d'information à l'usage des librairies, des bibliothécaires et des lecteurs, hiver-printemps $1994, \mathrm{n}^{\circ} 5$, p. 1.

2 Mentionnons notamment Monique Bosco, née à Vienne en 1927, vivant au Québec depuis 1948: Sara Sage, Montréal, HMH, 1986, Clichés, Montréal, $\mathrm{HMH}, 1988$; Rémémoration, Montréal, HMH, 1991; Gloria Escomel, née à Montevideo en 1941, au Québec depuis 1967: Fruits de la passion, Laval, Trois, 1988, Les eaux de la mémoire, Montréal, Boréal, 1994; Naïm Kattan, né à Bagdad en 1928, au Québec depuis 1954: Adieu Babylone, Montréal, La Presse, 1975, La fortune du passager, Montréal, HMH, 1989, Farida, Montréal, $\mathrm{HMH}, 1991$, La distraction, Montréal, HMH, 1994; Sergio Kokis, né en 1944 à Rio de Janeiro, au Québec depuis 1973: Le pavillon des miroirs, Montréal, XYZ éditeur, 1994; Dany Laferrière, né à Port-au-Prince en 1953, au Québec depuis 1978, mais qui se partage aujourd'hui entre Montréal et Miami en Floride: Comment faire l'amour avec un Nègre sans se fatiguer, Montréal, VLB éditeur, 1985, Cette grenade dans la main du jeune nègre est-elle une arme ou un fruit?, Montréal, VLB éditeur, 1993, Chronique de la dérive douce, Montréal, VLB éditeur, 1994; Mona Latif-Ghattas, née au Caire en 1946, au Québec depuis 1966: Le double conte de l'exil, Montréal, Boréal, 1990, La triste beauté du monde, Montréal, Éditions du Noroît, 1993; Marilù Mallet, née à Santiago du Chili en 1945, au Québec depuis 1973: Les compagnons de l'borloge pointeuse, Montréal, Québec/Amérique, 1981; Érnile Ollivier, né à Port-au-Prince en 1940, au Québec depuis 1965: Mère solitude, Paris, Albir Michel, 1983, La discorde aux cent voix, Paris, Albin Michel, 1986, Passages, Montréal, l'Hexagone, 1991; Marco Micone, né en 1945, au Québec depuis 1958: Addolarata, Montréal, Guernica, 1984, Le figuier enchanté, Montréal, Boréal, 1992; Ying Chen, née en Chine, vivant au Québec depuis 1989: L'ingratitude, Arles/Montréal, Actes Sud/Leméac, 1995; Hans-Jürgen Greif, né en Allemagne, vivant au Québec depuis 1969: L'autre Pandore, Leméac: 1990, Berbera, Montréal, Boréal, 1993, Solistes, Québec, L'Instant même, 1997: Régine Robin, née à Paris en 1939, au Québec depuis 1977: La Québécoite, Québec/Amérique 1983, réed. Typo, 1993 et L'immense fatigue des pierres, XYZ éditeur, 1996. 
28

esthétiques également très diverses. Leur point commun, au delà de cette désignation de néo-Québécois, est d'être des immigrés au Québec, pour certains en exil, d'écrire en français, - que le français soit leur langue maternelle, une deuxième langue ou une langue apprise sur le tard -, de se trouver au Québec dans son éternelle tension politique et identitaire, d'inscrire dans leurs œuvres une autre tension, celle du choc créé entre le pays d'origine, les souvenirs, la ou les langues, les senteurs, les paysages, la culture de là-bas et le pays d'accueil, en Amérique du Nord, mais avec une forte spécificité linguistique et culturelle, avec ses longs hivers et ses questionnements identitaires parfois indécodables. Ce travail de l'entre-deux, du dedans-dehors, de l'entrelangue, de l'errance et du regard venu d'ailleurs est le propre d'une écriture migrante qui joue un si grand rôle dans l'interrogation sur les nouvelles identités ${ }^{3}$. Longtemps regroupés dans le magazine culturel Vice Versa sous la direction de Lamberto Tassinari (est-ce tout à fait un hasard si la revue n'existe plus, de nouvelles subventions lui ayant été refusées tant à l'échelon fédéral qu'au niveau provincial?), certains écrivains néo-québécois forcent les portes de l'institution littéraire dans leur recherche d'un renouvellement des formes de l'écriture, des structures narratives, des questionnements existentiels, de l'imaginaire social de la société québécoise. Écritures hybrides, profondément américaines (au sens continental), québécoises et de langue française, éminemment transculturelles, elles s'inscrivent dans le devenir postmoderne des sociétés vouées plus que jamais à la pluralité culturelle.

Il se peut du reste que tout cela ne soit qu'une position transitoire, qu'un jour écrivains "néo" et écrivains dits "de souche" se rejoignent sans distinction d'origine. Rien que des écrivains, rien que de l'écriture. Le plus grand poète de l'exil au Québec n'est-il pas Jacques Brault? On se prend à rêver sur le dépassement des frontières et des appellations.

On est loin d'en prendre le chemin, me semble-t-il. Dans une plaquette publiée chez Fides, Monique LaRue met en scène un collègue, un écrivain québécois pris dans le ressentiment du succès de certains auteurs immigrants. "Cet écrivain n'avait pas prisé, par exemple, les multiples honneurs échus l'an dernier au seul

3 On lira à ce sujet Sherry Simon, Le trafic des langues, Montréal, Boréal, 1994. 
Sergio Kokis, ni les succès récents de Ying Chen ou de David Homel " ${ }^{4}$. Ce collègue lui expliquait la raison fondamentale de son ressentiment:

As-tu remarqué, me demandait-il, qu'une génération toute récente d'écrivains immigrants écrit des œuvres qui n'ont rien à voir avec ce qu'on a toujours appelé la littérature québécoise, des œuvres qui ne s'inscrivent d'aucune manière dans son histoire, dans la logique de son développement, qui ne poursuivent pas sa recherche d'identité, ne reprennent pas son réseau de références, sa dynamique intertextuelle, son imaginaire, qui n'intègrent à leur culture aucune des caractéristiques linguistiques issues de la démarche stylistique propre à la littérature québécoise, rien en somme de ce qui fait sa singularité au sein des littératures de la francophonie? ${ }^{5}$

Il ajoutait encore, comme pour mieux marquer son propos:

Ne trouves-tu pas, me disait-il encore, qu'il serait aberrant que ces écrivains dont l'œuvre ne se rattache ni par le contenu ni par la forme ni par le cadre au discours de notre littérature, soient autorisés à représenter la littérature québécoise à l'étranger, dans les colloques...? ${ }^{6}$

Monique LaRue avoue son malaise devant de tels propos. Derrière la hargne se dissimule, dit-elle, une vraie interrogation. Cette fois, c'est elle qui parle et assume son discours:

Notre littérature a jusqu'à maintenant été l'expression d'un monde commun, d'une expérience commune et relativement homogène, et nous ne nous sommes pas souvent demandé ce qu'était un écrivain québécois. $\mathrm{Si}$, politiquement, nous ne pouvons maintenant penser notre société que comme un monde hétérogène, pluriel, divers et cosmopolite, alors, sur le plan littéraire, quelle sera cette littérature québécoise? Parlera-t-on encore de littérature nationale? Comment penser la greffe de cette littérature telle qu'elle existe jusqu'à ce jour, avec la littérature telle que la conçoit l'autre ou une littérature autre, inconnue, à inventer? La diversité de perspectives forme-t-elle encore "une" littérature, une littérature spécifique parmi d'autres littératures distinctes, ou aurons-nous autant de littératures que de groupes ethniques? ${ }^{7}$

4 Monique Larue, L'arpenteur et le navigateur, Montréal, Fides et Cétuq, 1996, p. 7.

5 Ibid., p. 8.

6 Ibid., p. 8.

7 Ibid., p. 11 
30

Il est vain de se demander si l'écrivain "ethniciste", pétri de ressentiment, est un collègue "réel" ou s'il est constitué en personnage pour personnifier les démons qui ne cessent d'habiter l'écrivain québécois armé des meilleurs sentiments mais néanmoins assailli par des interrogations. Monique LaRue fustige ce discours tout en faisant état de ses propres doutes. La littérature québécoise, dit-elle, est constituée à la fois d'arpenteurs qui marquent et mesurent le sol, qui en prennent possession, se l'approprient (encore que le fameux K, arpenteur, héros du Château de Kafka que Monique LaRue convoque dans son texte sans aller jusqu'au bout de sa remarque, ne pourra jamais entrer dans le château, tout arpenteur qu'il soit), et des navigateurs, des nomades. Les deux entités constituent la littérature québécoise, parfois les écrivains eux-mêmes sont clivés entre leur part "arpenteur" et leur part "navigateur". Ce qu'il faut éviter absolument, nous dit Monique LaRue, c'est ce qu'elle appelle le syndrome Pravda. À la mort de Joseph Brodsky un obscur scribouillard russe écrivit la chose suivante:

Aux États-Unis d'Amérique est mort Joseph Brodsky [...] Moi j'ai été étonné de ce que les médias aient baptisé Joseph Brodsky "un grand poète russe" et je me suis posé la question: de quel droit? Je peux me tromper, mais il me semble pourtant que, pour porter ce titre, il ne suffit pas d'être lauréat du prix Nobel. On le sait, le poète en Russie est plus qu'un poète! Brodsky a-t-il mérité un tel titre? Aurait-il dans ses vers chanté la Russie? Ou bien prononcé de bonnes paroles sur le peuple russe? Je ne me rappelle rien de tel... Pouchkine et Essenine ont chanté tout cela et c'est pour cela qu'on les appelle "grands poètes russes". Mais Bodsky, dans le meilleur des cas, on peut l'appeler de "langue russe" et encore avec réticence puisque ces dernières années, il écrivait de plus en plus en anglais et qu'on l'enterrera non pas à Saint-Petersbourg mais à Venise. Quel "Russe" est-il donc? Peut-être que le mieux serait d'appeler Brodsky " grand poète juif". N'a-t-il pas dit: "je suis juif cent pour cent. On ne peut pas être plus juif que moi»? Les Juifs n'y trouveront rien à redire. Les Russes encore moins. ${ }^{8}$

Il y a quelques années, à l'institut Goethe je participais à une soirée avec des écrivains migrants de toutes nationalités. Je me

8 Ibid., p. 29. Monique LaRue a raison de faire suivre ce passage rapporté du journal russe de "Que de mesquineries". Elle a raison de souhaiter que la société québécoise puisse échapper à des semblables mesquineries. Scrutons l'horizon! 
souviens, en particulier, d'écrivains d'origine turque écrivant en allemand, vivant en Allemagne. Ils m'avaient frappée. La question. qui animait les débats, insolite, mais éclairante, était: quelle est votre nationalité littéraire?

Rien de moins évident que cette question d'apparence oxymorique. Il y a les réponses de type sociologique qui sont peutêtre les plus faciles à saisir: où sont vos maisons d'édition, qui vous fait signer un contrat et bien entendu dans quelle langue écrivez-vous, ce qui ne dit pas le tout de l'assise sociologique mais une grande part de cette première vérité. Pour un certain nombre d'écrivains du reste, même sur le plan sociologique, rien n'est simple car on peut écrire dans deux langues et relever de deux champs littéraires, ou écrire dans une seule langue mais relever tout de même de deux institutions différentes.

Sur le plan de l'identité vécue par l'écrivain, c'est autre chose. On aura toutes sortes de réponses. À quelle nationalité faire allégeance sur le plan littéraire? Comment désigner la nationalité de l'auteur? Un écrivain français? Un écrivain québécois? Un écrivain canadien d'expression française? Un écrivain juif? Un écrivain juif-français? Un écrivain juif québécois? Un écrivain juif canadien? Un écrivain juif allophone d'origine française? Un écrivain montréalais? On n'en finirait pas. À moins d'avoir sur le plan de la définition identitaire une fixation très forte, les choses sont très compliquées.

Tout cela ne dit rien d'une autre strate à prendre en considération lorsqu'on a affaire à des écrivains, c'est ce qui travaille leur texte sur ce plan précis, ce qu'ils inscrivent précisément d'une place impossible, l'entre-deux, les allers et retours identitaires qui sont les leurs. Quel est le narrataire implicite pour qui ils écrivent? Là résiderait un semblant de réponse à la question. Qui peut comprendre le texte, qui a le savoir implicite pour décoder les messages culturels du texte? Si j'écris au Québec comme je le fais pour tout ce qui concerne la fiction et que je parle en permanence du Paris des années cinquante, du Paris d'avant les grandes opérations immobilières, bien entendu je peux toucher les gens, produire toutes sortes d'effets esthétiques. Tout cela est bien connu. On n'est pas enfermé dans sa culture ni dans sa mémoire. Sinon, la traduction ne fonctionnerait pas. Je n'ai pas besoin de connaître l'aristocratie terrienne russe ni l'armée tsariste pour aimer Anna Karénine de Tolstoï. Cependant, le lecteur implicite qui habite mon texte, celui à qui je demande 
32

inconsciemment une complicité immédiate, celui qui pour moi peut partager toutes les allusions même les plus ténues, une date, un nom de rue, une chanson, un café, ce lecteur implicite ici sera français du type "je me souviens" de Georges Perec. Mais dans le même livre, me tournant tout à coup vers un autre personnage, j'évoque l'histoire juive et l'épisode du faux messie: Shabbatai Zevi, et peu à peu mon lecteur implicite se transforme, il est juif. À un autre moment enfin, c'est le débat autour des problèmes du Québec qui prend la première place, et là, mon lecteur implicite est québécois. La littérature est souvent un débat avec ses multiples identités. C'est pourquoi on ne peut répondre à cette question. Il n'y a pas de passeport ou de visa littéraire. On l'aura deviné, il n'y a pas pour moi de "nationalisme" innocent, ni les petits, les obscurs, les sans-grades, ni les grands, les vraiment menaçants, les "qui-ont-vraiment-fait-leurs-preuves", pas plus qu'il n'y a de "domination" innocente même tapie dans les sourires des personnages des heureux Disneyland. La littérature est un lieu spécifique qui tout en inscrivant une thématique qui peut être celle de la femme, voire l'écriture féminine, tout en inscrivant le fait minoritaire, voire le fait ethnique de façon plus ou moins spécifique, est en même temps le lieu qui problématise toutes les appartenances et toutes les identités, le lieu des expérimentations imaginaires et des interrogations sur ses propres appartenances. Qu'est-ce qu'être femme dans le texte littéraire? Comment définir le féminin dans l'écriture? Qu'est-ce qu'être "ethnique"? Il faut sortir des modèles binaires et voir ce qui fragilise la maîtrise, qu'elle soit idéologique, identitaire ou langagière. Il en va de même pour l'ethnicité. Toute identité trop fortement définie fût-elle minoritaire - est un enfermement. À mes yeux, seule l'œuvre littéraire a la force aujourd'hui de rendre sa pleine valeur au fantasme. Lors de cette soirée, je me suis définie comme une allophone d'origine française, au milieu des fous rires, bien entendu. Je crois que font partie du nécessaire dévoiement des cultures le blasphème, le sacrilège ou tout simplement l'ironisation, la parodie, la mise à distance de l'origine. Nous sommes nombreux à tenter d'écrire dans une langue alors qu'on rêve d'une autre langue mais que la seconde est barrée, ce que j'ai appelé dans un des mes livres "la langue perdue"?. Oublier la

9 Régine Robin, Le deuil de l'origine. Une langue en trop, la langue en moins, Paris, Presses de l'Université de Vincennes, 1993. 
langue paria? Freud s'invente une langue universelle, et Kafka, dans son allemand de papier, fragilise la langue par la fragilisation du référent, l'altération des légendes, la blessure ou la cassure, le devenir chose ou le devenir animal, le statut de la noncompréhension du langage, le destin des lettres et des dossiers ou celui de la communication au Château, le destin de la communication au Château, le langage des gestes et le langage des lèvres dans Le procès. Elias Canetti retrouve brutalement l'oralité de son grand-père sur le marché de Marrakech, et Georges Perec par la pratique du palindrome écrit à la fois en français et en yiddish ou en hébreu, plus exactement, le double sens de l'écriture palindromique (de gauche à droite et de droite à gauche) lui fait simuler le sens de l'écriture hébraïque dans le français.

De là, pour moi, le recours à la fausse biographie, à la fausse autobiographie, au regard sur le passé pour lui trouver un sens nouveau, la quête qui a fait le deuil de la synthèse, le roman proprement dit, l'autofiction, la transformation du biographique en fiction. Le tout par bribes, par touches qui inscrivent du biographique de façon tenue. Mais je m'empresse d'ajouter que mon roman n'est pas autobiographique. Sur le plan politique, par exemple, la logique narrative m'entraîne à dire qu'" elle", le personnage principal, ne sait pas comment elle aurait voté au premier référendum. L'incertitude de la narratrice lui permet toutes sortes de développements, de réflexions sur le problème. Si j'avais dit qu'elle avait voté "non", j'aurais clos la discussion. Or dans la fiction, je voulais laisser ouverts tous les chemins possibles. De toute façon, ce sont des suppositions. Ni l'auteur (moi), ni la narratrice, ni le personnage n'avaient à l'époque le droit de vote, elles pouvaient toujours divaguer. Moi, Régine Robin, j'aurais voté "non" en 1980 comme j'ai voté "non" en 1995. Mais il est important sur le plan de la fiction de laisser toutes les portes ouvertes afin de pouvoir laisser le champ libre à l'expérimentation identitaire, à la méditation, à la réflexion.

Mon personnage est fictif. Il n'est qu'un support narratif. Je le déplace dans trois quartiers différents de Montréal. Et puis, ce personnage est d'origine ukrainienne; elle a une tante qui avant d'immigrer à Montréal était en Ukraine et qui est passée par Londres. J'ai inventé tous les détails, je n'ai jamais eu la moindre tante ici. Le récit contient des éléments autobiographiques, mais qui n'ont pas été nécessairement attribués au personnage principal. Ils sont complètement disséminés. Certains éléments sont 
34

portés par le personnage de l'écrivain qui tente de faire son livre sur Shabbatai Zevi, d'autres par les personnages qu'elle croise. Il y a, par exemple, ce New-Yorkais qu'elle rencontre qui peut faire penser à mon mari, lequel n'est pas new-yorkais. Les éléments autobiographiques sont transformés, pris dans une trame narrative qui les déforment. On a pensé que mon livre était autobiographique. Mais non! L'autobiographie, si autobiographie il y a, est intellectuelle, spirituelle, absolument pas factuelle.

On me permettra d'ajouter que je trouve ce livre d'une étonnante actualité, un peu comme s'il avait été écrit l'an dernier. Bien sûr, les Steinberg n'existent plus, ils ont été remplacés par des Métro, le prix des maisons que je trouvais faramineux est devenu dérisoire, ce sont les phénomènes naturels du vieillissement d'une société. Au niveau des analyses cependant, des rapports inter-culturels, du mal-être des fameux "ethniques", rien, ou pas grand-chose, n'a bougé.

Si, peut-être, pour moi, la familiarisation progressive avec la langue anglaise. Chacun sait qu'on n'a jamais appris sérieusement l'anglais en France. Je le regrette.

Quand je suis arrivée ici, je lisais mal l'anglais, je le comprenais mal et je le parlais très mal. Paradoxalement, ce que le Québec m'aura donné, c'est l'anglais. Il y a vingt ans, j'ai appris sérieusement l'anglais, je suis même allée suivre des cours à l'Université McGill. En regardant tous les jours la têlévision canadienne anglaise et les chaînes américaines, en habitant à l'ouest de la ville, en allant souvent à New York, en étant souvent invitée dans des universités américaines - NYU, Harvard, Stanford, Chicago - je me suis mis l'anglais "dans l'oreille".

Je peux à présent voir des films anglais, américains ou australiens sans sous-titres. Il me reste néanmoins un accent français très reconnaissable et une gaucherie indépassable mais l'anglais est devenu une de "mes" langues et $j$ 'en suis ravie. On admettra que ce n'est pas là une position classique, mais une position d'écrivain qui, s'il n'écrit que dans une langue (je suis attachée au français comme le bernard-l'ermite à sa coquille), est habité par d'autres en dialogue, en symbiose, en conflit.

La mode du "trans" est paraît-il passée. Comme si pour nous, ça avait été "une mode". Peur de la modernisation, repli sur les valeurs "nationales", peur de la dissolution des identités. Sale 
période! En particulier pour les écrivains! Qu'on lise en particulier le dernier livre de Serge Cantin ${ }^{10}$, on y trouvera toutes les problématiques que je récuse. De violentes diatribes contre Nadia Kouri, Esther Delile, Charles Taylor, une attaque contre l'écrivain Jacques Godbout, trop "ambigu" aux yeux de l'auteur, coupable d'avoir laissé entendre que Mordechaï Richler serait peut-être un des plus grands écrivains québécois. Serge Cantin écrit à ce propos:

Qu'est-ce qui cloche dans un tel raisonnement? Avant tout, c'est que Jacques Godbout gomme une réalité primordiale: la langue, pour ne retenir, comme unique critère d'appartenance à la société et à la culture québécoise, que le fait de résider sur le territoire de la province de Québec. Il est frappant en effet que le rapprochement qu'il [Godbout] établit entre Céline et Richler fait l'impasse sur une différence à mes yeux capitale, à savoir que l'écrivain français Céline écrivait en français alors que celui que nous devrions considérer comme le plus grand écrivain québécois, écrit, lui, en anglais. Détail de toute évidence négligeable aux yeux de Godbout qui préfère suivre la logique du dictionnaire... Léonard Cohen, qui lui aussi est né et a grandi à Montréal dans une famille juive, n'est pas et ne sera jamais un "chansonnier" québécois. Dois-je ajouter, que personnellement j'adore Cohen, bien que lui non plus, parait-il, n'ait pas été toujours tendre à l'endroit des Québécois? Mais, le fait est que comme tant d'autres anglophones montréalais, Cohen n'est pas et, à ma connaissance, n'a jamais eu l'intention de devenir Québécois, ou plutôt il n'est pas Québécois parce qu'il n'a jamais eu l'intention de le devenir... ${ }^{11}$

Moi, j'écris des romans, des récits, des nouvelles, des essais en français. Mais, pas plus que Richler ou Cohen, je ne suis "un écrivain québécois" aux yeux des écrits de Cantin. Je ne sais pas si j'ai le désir de le devenir, à lire ses propos... Je suis, je le répète, une allophone d'origine française.

Dans une note de son livre, Serge Cantin, en avouant sa perplexité à l'égard de la dénomination de "Francophones du Québec " utilisée par Fernand Dumont ${ }^{12}$, explicite de ce qu'il appelle "Québécois":

En gros son argument [à Fernand Dumont] est qu'il n'y a pas de nation québécoise, mais une nation française ou francophone,

10 Serge Cantin, Ce pays comme un enfant, Montréal, l'Hexagone, 1997.

11 Ibid., p. 85.

12 Fernand Dumont, Raisons communes, Montréal, Boréal, 1995. 
Tangence 59

36

nation majoritaire à l'intérieur de la société québécoise qui ellemême regroupe plusieurs nations: francophone, anglophone, autochtone, sans compter les allophones. Précision importante, j'en conviens, notamment en regard des conditions d'exercice de la démocratie dans un Québec souverain. Reste que l'adoption, au début des années 1960, de nom de "Québécois" par ceux qui, jusqu'alors, s'étaient appelés les Canadiens français, ou, avant, les Canadiens, fut et demeure, aujourd'hui encore, dans l'imaginaire collectif, le symbole d'une affirmation de soi et d'une prise de conscience politique de la domination et de l'aliénation séculaires de la nation française d'Amérique. J'avoue ici mon embarras. ${ }^{13}$

Sale période alors pour "les autres", les "néos", les...!

J'avais imaginé, il y a quelques années, lors d'un colloque international à Mendoza, en Argentine, un scénario hallucinant. Pour se débarrasser des écrivains, trouble-fête (voir Salman Rushdie ou Günther Grass), on les avait tous rassemblés dans un immense camp de concentration, version soft, quelque part en Patagonie, loin de tout, dans de grands espaces solitaires. Là, ils pouvaient continuer à écrire, voire à publier, sans que cela sorte des limites assignées, sans pouvoir franchir une clôture qui, pour ne pas être faite de barbelés, n'en était pas moins réelle. Ils continuaient du reste à écrire, mis à part le petit nombre en dépression constante qui ne pouvaient accepter la situation, et le plus petit nombre encore qui s'étaient suicidés au début de leur relégation. Le reste continuait à écrire en se querellant. On imagine le parti qu'on pourrait tirer de tels développements. Le plus intéressant concernait ce qui se passait en dehors de la Patagonie, de ce camp. Le monde continuait comme avant, avec ses guerres, ses préoccupations, et personne, absolument personne, ne s'était aperçu qu'il n'y avait plus d'écrivains, plus d'écriture, plus de fantasmes (on pouvait tous les réaliser), plus d'imaginaire créateur, plus de rêves (à quoi sert encore "le roman familial" si on peut programmer les enfants, voire les parents qu'on veut?). On ne s'était aperçu de rien. Un monde sans écrivains, sans écriture, et cela passait inaperçu à l'heure du prime time de la télévision.

Alors, au-dessus de la mêlée, l'écrivain? la "néo"? (toujours mal à l'aise avec les féminins: l'écrivaine), pas tout à fait, mais... La Québécoite rend compte de cette position. Ni dedans ni

13 Serge Cantin, op. cit, p. 178. 